\title{
A Sorting System for Aluminum Alloy Scrap Based on Laser-Induced Breakdown Spectroscopy
}

\author{
Mikio Kuzuya \\ Department of Electrical and Electronic Engineering, College of Engineering, Chubu University, Kasugai, Japan \\ Email address: \\ Kuzuya@isc.chubu.ac.jp \\ To cite this article: \\ Mikio Kuzuya. A Sorting System for Aluminum Alloy Scrap Based on Laser-Induced Breakdown Spectroscopy. Science Journal of Analytical \\ Chemistry. Vol. 7, No. 3, 2019, pp. 65-71. doi: 10.11648/j.sjac.20190703.11
}

Received: May 28, 2019; Accepted: July 3, 2019; Published: July 13, 2019

\begin{abstract}
A sorting system for aluminum scrap using laser-induced breakdown spectroscopy (LIBS) has been developed, which employs the sequential sample cells that are moved by the electric actuator in order to carry scrap pieces. A combination of a Q-switched Nd: YAG laser (30mJ, 10ns) with an objective lens having a long focal length of 600 mm were used to generate plasmas, and the emitted light was analyzed spectroscopically by a compact fiber-optic spectrometer having a CCD array detector. A pulse generator was constructed in order to synchronize the laser pulse, the CCD spectrometer and the electric actuator. The elemental composition of aluminum alloy scrap was determined by the calibration curves obtained by analyzing the standard aluminum alloy samples. The sorting algorithm based on the difference of the concentration of main additional elements of aluminum alloy was proposed. The developed LIBS sorting system was applied for the analysis of aluminum alloy samples and complete classification and separation of cast and wrought aluminum alloys into specific alloy groups was achieved.
\end{abstract}

Keywords: Laser-Induced Breakdown Spectroscopy (LIBS), Q-switched Nd: YAG Laser, Sorting, Aluminum Scrap, Cast and Wrought Alloys, Calibration Curve

\section{Introduction}

It is widely recognized that recycling of metal scrap will contribute to resource saving and energy conservation. For instance, the use of recycled aluminum requires as little as $5 \%$ of the energy needed for the production of primary aluminum and results in significant savings in energy consumption. Currently, in the case of aluminum recycling, most aluminum scrap is recycled via cascade recycling, in which different kinds of aluminum alloys are mixed and used as casting alloys resulting in downgrade recycling. If the separation of aluminum scrap into individual alloys is achieved, the down-cycling of aluminum scrap can be prevented and the recycling rate for aluminum can be improved significantly. Therefore, an analytical method for sorting of metal scrap is required.

Laser-induced breakdown spectroscopy (LIBS) [1-3] is one of the suitable sorting technologies for metal scrap, because of its capability of performing remote and fast multi-elemental analysis of any type of material without significant requirements of sample preparation in air at atmospheric pressure. Recently, the chemical analysis of aluminum alloys by LIBS has been studied extensively [4-6].

We have studied the emission characteristics of the laser-induced plasma to improve the analytical performance, and showed the optimum conditions for quantitative analysis for metal alloys [7-17]. In the previous work [18], the author investigated the applicability of LIBS for sorting of aluminum alloy scrap and showed that the developed LIBS system can analyze the elemental composition of each piece of scrap with a single laser shot, and classify both cast and wrought alloy scrap pieces into individual alloys by the calibration curves obtained using the method of an internal standard. However, the experiment was based on a static measurement setup. From the viewpoint of field-based and industry applications, it is required to combine the LIBS system with the mechanics of carrying the scrap pieces and sorting the analyzed pieces by alloy type.

In the present work, the author has developed an automatic sorting system for aluminum scrap using LIBS, which employs the sequential sample cells that are moved by the electric actuator in order to carry scrap pieces. The scrap piece is dropped at the position of an appropriate sorting bin by opening the bottom plate of the sample cell according to the 
corresponding sorting signal. The experiment was carried out under the condition obtained in the previous work. By using this LIBS sorting system, the determination of the elemental composition of aluminum scraps and the successful separation of cast and wrought aluminum alloys into individual groups were achieved.

\section{LIBS Sorting System}

In general, the LIBS sorter consists of a combination of the LIBS apparatus and a belt conveyor for carrying scrap pieces. In such a system, the precise measurement of the shapes and positions of the scrap is necessary in order to focus the laser pulse onto the surface of the moving scrap. Moreover, in order to compensate the laser defocus due to the difference of the height of each scrap pieces, a combination of a distance meter and autofocusing unit of the LIBS laser is needed. The system is therefore complicated and expensive.

To resolve the above problem, the electric actuator instead of the belt conveyor is used in order to carry the scrap pieces. The scrap pieces are placed in the sequential sample cells arrayed in a fixed distance which is moved by the electric actuator after the laser firing. In this method, because the scrap sample can be analyzed in a stationary state, the LIBS apparatus used in the previous work can be almost utilized as it is.

Figure 1 shows a schematic diagram of the LIBS sorting system developed in this work, which is basically the same as that used in the previous work, except for changing the spectrometer and the sample stage. A Q-switched Nd: YAG laser (Lumonics, HY-400) was operated at the fundamental wavelength of $1064 \mathrm{~nm}$ with a pulse duration of $10 \mathrm{~ns}$. The laser pulse energy was set to $30 \mathrm{~mJ}$ at which the emission spectrum with a high line intensity and line-to-background ratio could be obtained [18]. The laser beam was irradiated onto the sample surface with an objective lens having a long focal length of $600 \mathrm{~mm}$. The light emitted from the laser-induced plasma is collected almost collinearly to the incident laser beam by a collimator lens which is positioned at $150 \mathrm{~mm}$ above the bottom of the sample cell and analyzed spectroscopically by a compact fiber-optic spectrometer (Ocean Optics, Maya2000 Pro) having a CCD array detector. The spectrometer covers the spectral range of 199-423 nm with a resolution of $0.1 \mathrm{~nm}$. The spectral data obtained is transferred to the computer via USB and processed by the software which has developed in our laboratory in order to analyze the spectrum and classify aluminum alloys into individual groups.

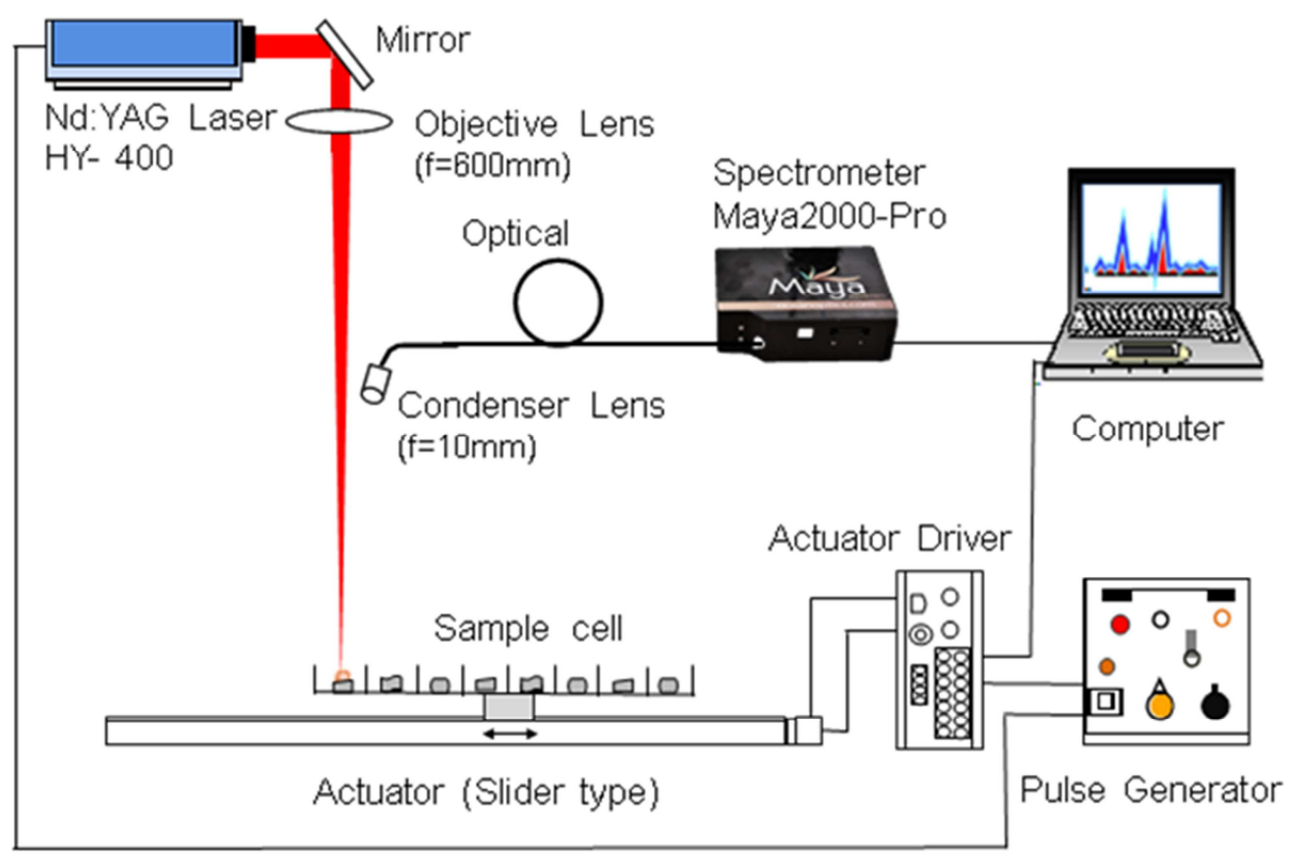

Figure 1. Schematic diagram of the Laser-Induced Breakdown Spectroscopy (LIBS) sorting system.

In this experiment, seven sample cells (cell size $40 \mathrm{~mm} \times 40$ $\mathrm{mm} \times 20 \mathrm{~mm}$ ) are mounted on the electric slider (Oriental Motor, EZSHM) which can be moved at the maximum speed of $1800 \mathrm{~mm} / \mathrm{s}$. Timing of the LIBS laser, the electric slider and the CCD spectrometer are controlled by using a pulse generator which was constructed in our laboratory.

Figure 2 shows the timing diagram of each apparatus when the laser is operated at a repetition rate of $10 \mathrm{~Hz}$. The spectrometer starts to measure the spectrum at a time delay of
$3.1 \mathrm{~ms}$ (CCD initialization time) after a trigger pulse in synchronization with a clock pulse of $10 \mathrm{~Hz}$. The laser is fired at a time delay of $5 \mathrm{~ms}$. The integration time for measuring the spectrum is set to $10 \mathrm{~ms}$. After the measurement is completed, a trigger pulse with a time delay of $30 \mathrm{~ms}$ is sent to the actuator drive and the electric slider moves by $40 \mathrm{~mm}$ which corresponds to the distance of each sample cell. This time sequence is repeated every analysis. 


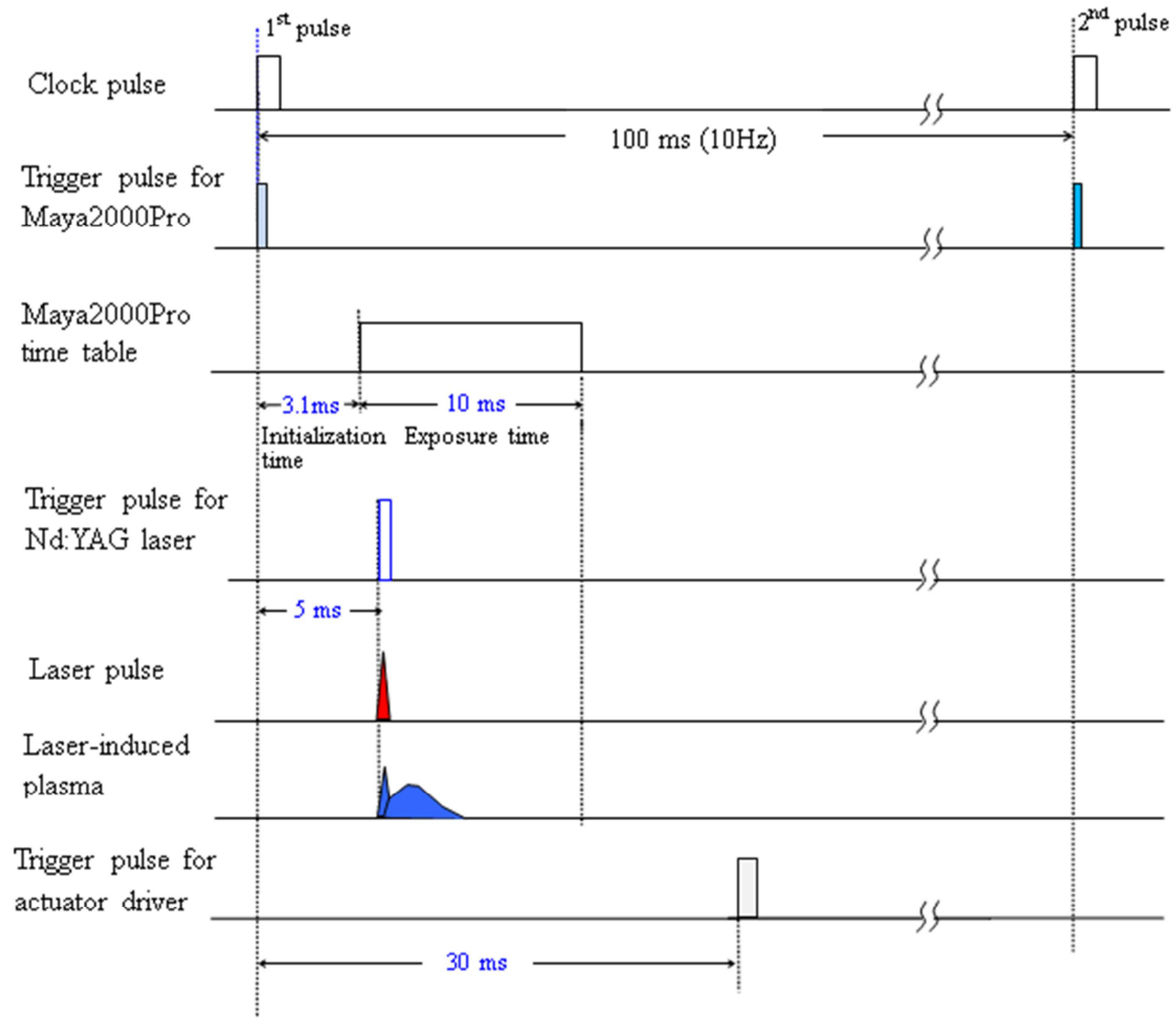

Figure 2. Timing diagram of each apparatus of the LIBS system.

The samples used in quantitative analysis were the standard aluminum alloy samples (AC2BNB, AC5AB22, ADC10NH1, ADC10FM, ADC12NP, ADC12NP, 3SNDC2, 52SNDC3, $6070 \mathrm{NDC} 1$, and 7N01NDC), certified and supplied by Nippon Light Metal Co., Ltd, and five principal alloying elements of $\mathrm{Si}, \mathrm{Cu}, \mathrm{Mn}, \mathrm{Mg}$, and $\mathrm{Zn}$ were analyzed in order to construct the calibration curves. Table 1 shows the compositions of principal alloying elements of the standard aluminum alloy samples.

Table 1. Chemical composition of standard aluminum samples (wt\%).

\begin{tabular}{llllll}
\hline Sample & Si & Cu & Mn & Mg & Zn \\
\hline AC2BNB & 6.92 & 2.60 & 0.05 & 0.06 & 0.81 \\
AC5AB22 & 0.60 & 3.99 & 0.35 & 1.80 & 0.14 \\
ADC10NH1 & 8.04 & 3.73 & 0.10 & 0.10 & 0.42 \\
ADC10FM & 10.82 & 4.36 & 0.50 & 0.66 & 0.11 \\
ADC12NP & 12.04 & 2.46 & 0.39 & 0.26 & 1.46 \\
3SNDC2 & 0.40 & 0.05 & 1.26 & 0.04 & 0.05 \\
52SNDC3 & 0.15 & 0.05 & 0.05 & 2.46 & 0.02 \\
6070NDC1 & 2.29 & 0.14 & 0.54 & 0.45 & 0.93 \\
7N01NDC & 0.11 & 0.06 & 0.61 & 1.50 & 4.26 \\
\hline
\end{tabular}

\section{Results and Discussion}

\subsection{Calibration Curves}

At first, in order to find the optimum analytical line for the calibration curve, the spectral lines of the principal alloying elements were investigated because the spectrometer was changed. Figure 3 shows typical emission spectrum of aluminum alloy sample obtained by a Maya200 Pro spectrometer. The sample used is a standard aluminum alloy sample (ADC12NP). As the best analytical lines for each alloying element, the spectral lines of Si I $288.1 \mathrm{~nm}, \mathrm{Cu}$ I $324.75 \mathrm{~nm}, \mathrm{Mn}$ I $403.0 \mathrm{~nm}, \mathrm{Mg}$ I $285.2 \mathrm{~nm}$, and Zn I $206.1 \mathrm{~nm}$ were selected because these spectral lines have reasonable intensities and are free of interference from other elements, as shown in Figure 3.

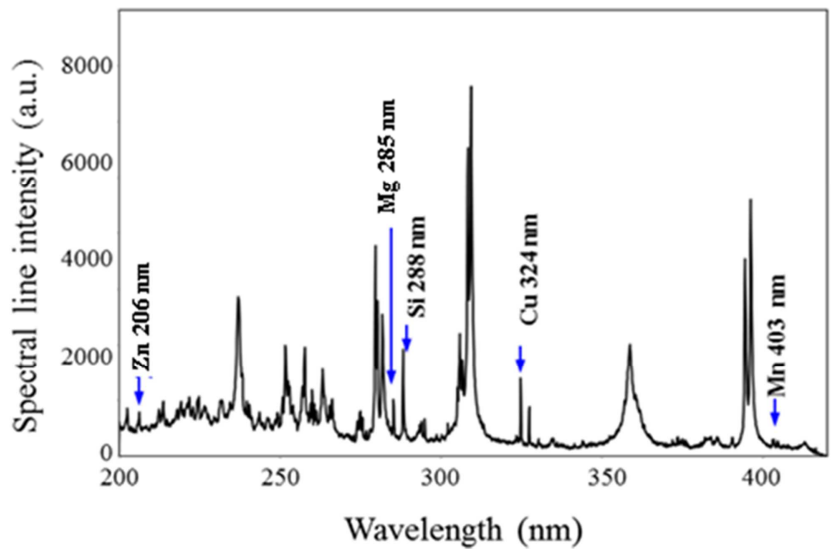

The sample used is a standard aluminum alloy sample (ADC12NP).

Figure 3. Typical emission spectrum of aluminum alloy sample obtained by a Maya200 Pro spectrometer. 

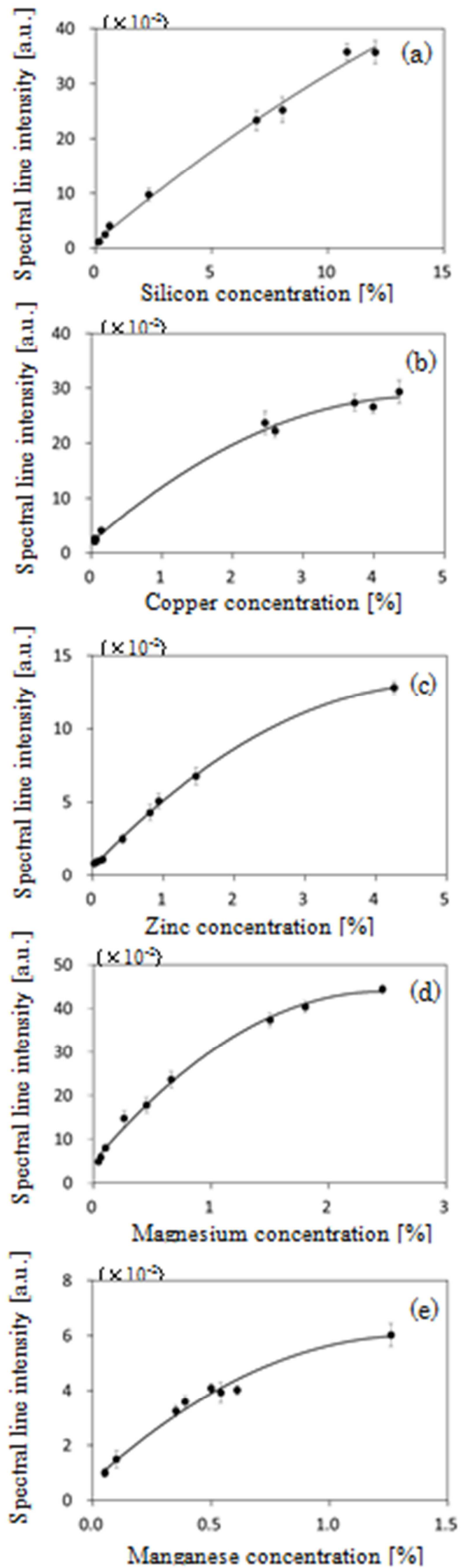

Figure 4. Calibration curves for the analysis of (a) silicon (Si I $288.1 \mathrm{~nm}$ ), (b) zinc (Zn I $334.5 \mathrm{~nm}$ ), (c) magnesium (Mg I $383.8 \mathrm{~nm}$ ) and (d) manganese (Mn I $403.0 \mathrm{~nm}$ ) in standard aluminum alloy samples.
The standard aluminum alloy samples listed in Table 1 were analyzed for the principal alloying elements and the calibration curves were constructed by using the method of internal standard, because it has been indicated in the previous work that this method can compensate the variations in the spectral line intensity due to the changes in the sample height. The results are shown in Figure 4. The Intensity of each analytical line was normalized to the intensity of Al I 396.15-nm line as the internal standard. Spectral intensities were obtained by averaging ten measurements and the standard deviation is shown with an error bar. The calibration curves show a curvature for higher concentrations due to the effect of self-absorption of analysis lines by the plasma. Therefore, the calibration curves were approximated by the quadratic function which was obtained by fitting the experimental data, in order to calculate the concentration of element from the measured spectral intensity.

\subsection{Classification of Aluminum Alloys}

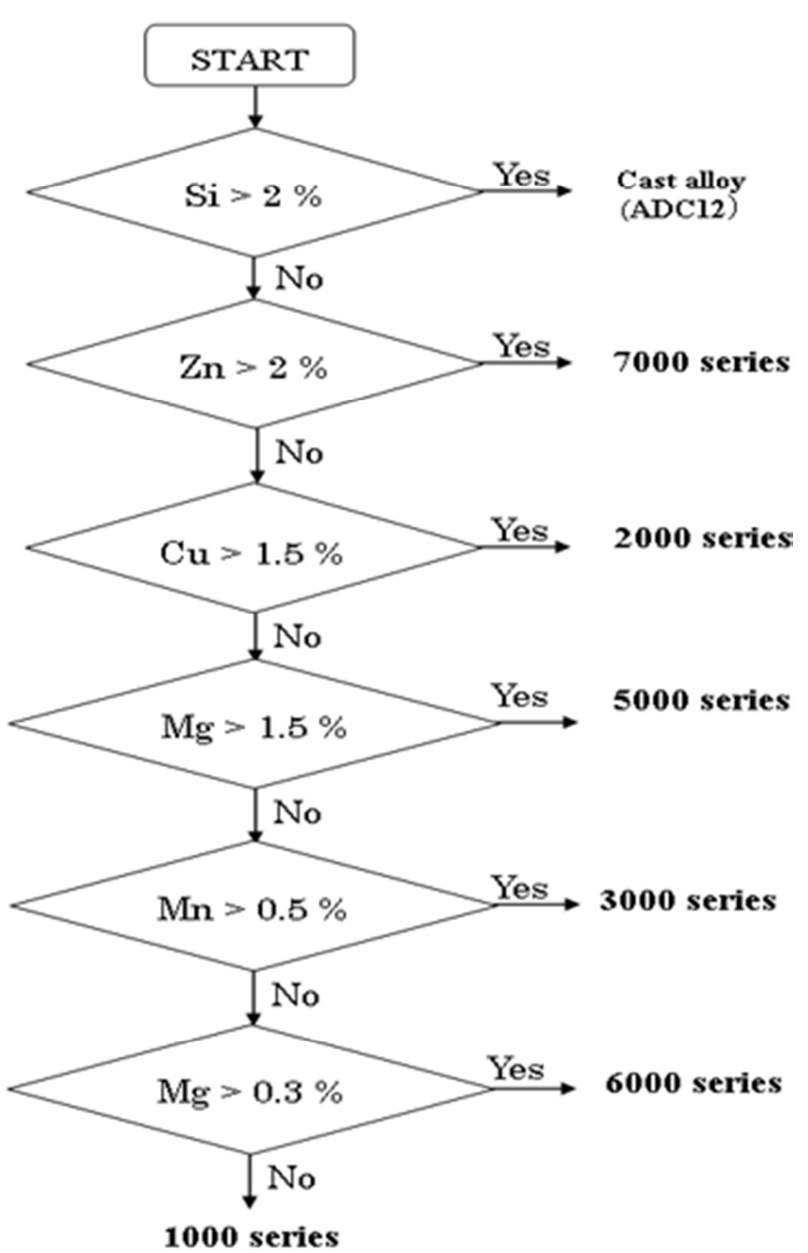

Figure 5. Classification method of aluminum cast and wrought alloys into seven aluminum groups.

Aluminum alloys are classified in two major groups of wrought and cast alloys, and wrought alloys can be divided into eight series from 1000 to 8000 depending on the principal alloying element. However, even when wrought alloy scraps 
have been classified, individual alloys can have relatively widely varying elemental compositions. Therefore, the separation of aluminum scrap into individual class requires compositions with relatively broad specification limits on the principal alloying elements and other impurities. Table 2 shows the compositional limits of recycled aluminum which were adopted for sorting aluminum scrap in the previous work. By taking account of reusing recycle aluminum scrap, aluminum alloys are classified into cast alloy and five wrought alloy series $(2000,3000,5000,6000,7000)$, and cast alloy and the 4000 series of wrought alloy are reused for cast alloy (ADC12).

It is found from the compositional limits of Table 2 that aluminum alloys can be sorted by checking a difference of the concentration of main additional element, according to the order of sorting elements as shown in Figure 5. In this work, this algorithm was adopted to sort aluminum alloys because the effectiveness of this sorting method was confirmed in the previous work. It should be noted that the segregation of the 1000 series of the wrought alloy is newly added to the sorting algorithm in Figure 5.

Table 2. Aluminum alloy groups and concentration limits of the alloying element.

\begin{tabular}{|c|c|c|c|c|c|c|c|c|}
\hline \multirow{2}{*}{ Sample } & & \multicolumn{7}{|c|}{ Alloying element (\%) } \\
\hline & & $\mathbf{S i}$ & $\mathbf{C u}$ & Mn & Mg & $\mathbf{Z n}$ & $\mathbf{F e}$ & $\mathbf{T i}$ \\
\hline \multicolumn{2}{|c|}{ Cast alloy (ADC12) } & $>=2.0$ & - & $<=0.6$ & - & $<=3.0$ & $<=1.3$ & $<=0.3$ \\
\hline \multirow{5}{*}{ Wrought alloy } & 1000's & $<=0.05$ & $<=0.25$ & $<=0.05$ & $<=0.05$ & - & $<=0.4$ & $<=0.1$ \\
\hline & 2000’s & $<=0.5$ & $1.5-5$ & $<=0.9$ & $<=1.8$ & $<=0.2$ & $<=0.7$ & $<=0.1$ \\
\hline & 3000’s & $<=0.8$ & $<=0.2$ & $0.5-1.5$ & $<=1.3$ & $<=0.2$ & $<=0.7$ & $<=0.1$ \\
\hline & 5000’s & $<=1.3$ & $<=0.1$ & $<=0.1$ & $1.5-2.8$ & $<=0.2$ & $<=0.4$ & $<=0.1$ \\
\hline & 6000’s & $<=0.6$ & $<=0.1$ & $<=0.1$ & $0.5-1.2$ & $<=0.2$ & $<=1.0$ & $<=0.1$ \\
\hline
\end{tabular}

The classification experiment was carried out in order to confirm the operating performance of the LIBS sorting system. The sample used are seven kinds of aluminum alloys of pure aluminum (1000 series), 24SNDC2 (2000 series), 3SNDC2 (3000 series), AC2BNB (cast alloy), 52SNDC3 (5000 series), 6063 alloy scrap (6000 series) and 7075NDC2 (7000 series), and each sample was placed in a sample cell in turn, as shown in Figure 6. The laser was operated at the repetition rate of $10 \mathrm{~Hz}$. Each sample was analyzed two times and the classification results are shown in Table 3 with the concentration of the principal alloying element. A pure aluminum was classified into the 1000 series and other aluminum alloys were classified into individual alloy series according to the principal alloying element. It was confirmed from this result that the developed LIBS sorting system can be utilized for sorting of aluminum alloys.

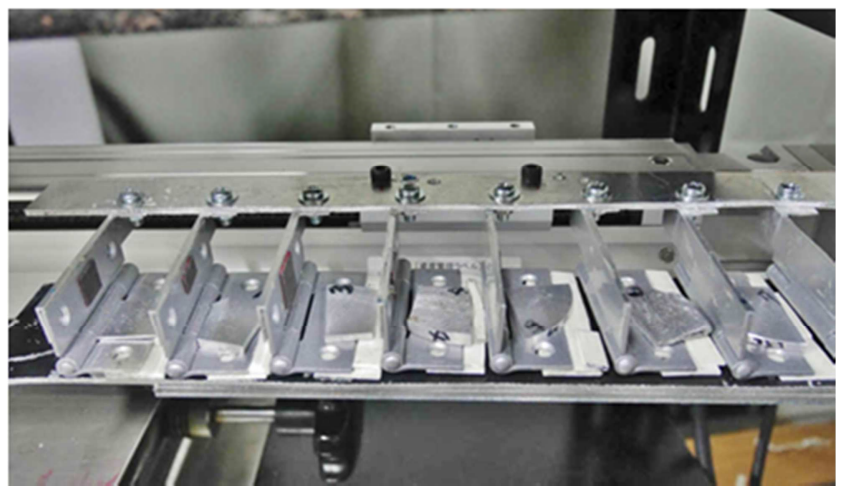

Figure 6. Aluminum alloy samples set in the sequential sample cells.

Table 3. Concentration of major element of aluminum alloy samples and sorting results by LIBS.

\begin{tabular}{llll}
\hline Sample & Major element & Conc. (\%) & Sorting result \\
\hline \multirow{2}{*}{ Pure aluminum } & - & - & 1000 's \\
& - & - & 1000 's \\
24SNDC2 & $\mathrm{Cu}$ & 4.5 & 2000 's \\
& $\mathrm{Cu}$ & 4.5 & 2000 's \\
3SNDC2 & $\mathrm{Mn}$ & 1.3 & 3000 's \\
& $\mathrm{Mn}$ & 1.3 & 3000 's \\
AC2B & $\mathrm{Si}$ & 6.4 & ADC12 \\
& $\mathrm{Si}$ & 7.0 & ADC12 \\
52SNDC3 & $\mathrm{Mg}$ & 2.4 & 5000 's \\
& $\mathrm{Mg}$ & 2.4 & 5000 's \\
6063 & $\mathrm{Mg}$ & 0.5 & 6000 's \\
$7075 N D C 2$ & $\mathrm{Mg}$ & 0.6 & 6000 's \\
\hline
\end{tabular}

\subsection{Separation of Aluminum Alloys}

In view of the application of the LIBS sorter at a recycling plant, a mechanical device that separates the classified scrap into an appropriate sorting bin is required. Figure 7 shows the photograph of the separating device constructed in this work.
The separating device consists of a combination of an openable bottom plate of the sample solenoid. A board for supporting the bottom plate of the irradiation position (A in Figure 7), and seven slide bars are arranged on the left side and the bar from the first to the seventh is assigned to the alloy from 1000 series to 7000 series, respectively. It is of note that 
the fourth bar is also assigned to the cast alloy. The sample cell moves from the right to the left synchronously with a laser pulse. When the sample cell comes to the position of the slide bar corresponding to the alloy series of the scrap piece in the cell, the electromagnetic solenoid is activated and the slide bar is pulled out to open the bottom plate of the sample cell, resulting in dropping the scrap piece. The separating signal is sent from the computer to a control circuit which drives the electromagnetic solenoid through the USB-IO unit (Km2Net Inc.) For example, when the scrap piece is classified into the 3000 series, the electromagnetic solenoid of the third slide bar will be activated after 3 laser shots.

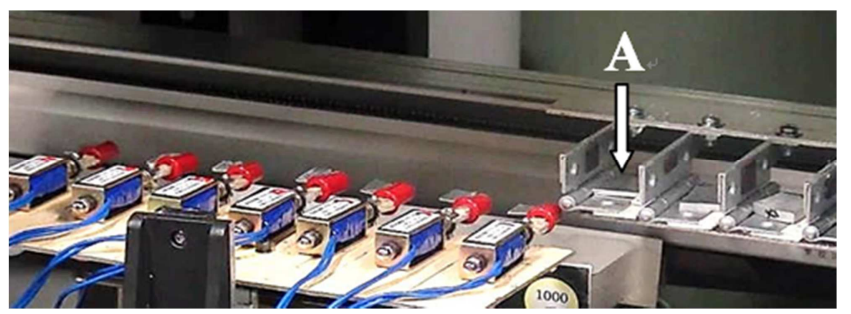

The laser is irradiated at the position A.

Figure 7. Photograph of sequential sample cells and separating device.

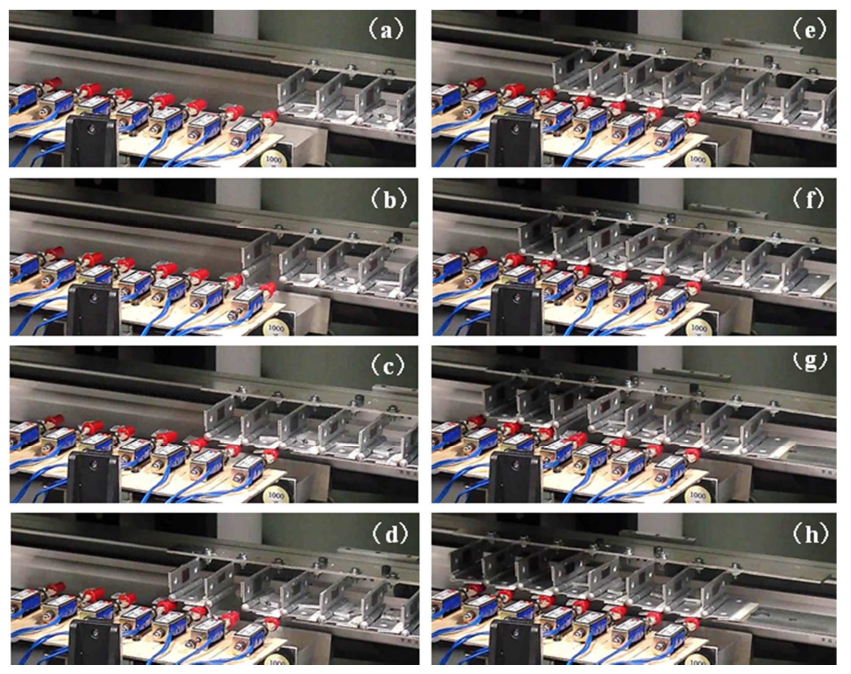

Figure 8. A series of photographs showing the separation of three samples of a pure Al metal, a standard sample of $24 S N D C 2$ and a wrought scrap alloy.

In order to test the movement of the separating device, a sorting experiment of 3 samples of a pure Al metal, a standard sample of 24SNDC2 and a wrought scrap alloy, which were set in the 1st, 2nd and 3rd sample cells were carried out. Figure 8 (a)-(h) are a series of photographs which show the sorting of each sample. A pure Al metal was classified at the 1st laser shot (a) and dropped in the 1st bin (1000 series) at the timing of the 2nd laser shot (b). A standard sample of 24SNDC2 was also classified at the 2nd laser shot (b) and dropped in the 2 nd bin (2000 series) at the timing of the 4 th laser shot (d). Finally, a wrought scrap alloy classified at the 3rd laser shot (c) was dropped in the 4th bin (cast alloy) at the timing of the 7 th laser shot $(\mathrm{g})$. It was confirmed from the results that the constructed separating device could operate normally.

Recently, Campanella et al. [6] published the paper on classification of wrought aluminum alloys by artificial neural network (ANN) evaluation of LIBS spectra from aluminum scrap samples. They analyzed 39 real aluminum scrap samples which belonged to four wrought series $(3000,5000,6000$, 7000) and showed that the percentage of samples correctly and wrongly classified was $74 \%$ and $15 \%$, respectively, while $11 \%$ of the samples were not classified. They also reported that this result could be satisfactory in the case of real aluminum alloys classification realized with single-shot measurement.

In this work, aluminum alloy scrap was classified by checking the difference of the concentration of the main additional element which was determined by the calibration curve. In the previous work, 121 real aluminum scraps ( 7 cast alloys and 114 wrought 6000 series alloys) were analyzed and complete classification was achieved.

The emission intensity of the LIBS spectrum was strongly influenced by the laser energy, and higher laser energy did not bring about high emission intensity, due to the effect of gas breakdown $[9,16]$. In the previous paper, the effect of the laser energy on the emission intensity was investigated, and the maximum emission intensity and the highest line-to-background ratio were obtained at the laser pulse energy of around $30 \mathrm{~mJ}$. Hence, one of the cause for the low percentage of correct classifications obtained by Campanella et al. may be the use of the higher laser energy of $60 \mathrm{~mJ}$ compared with $30 \mathrm{~mJ}$ in the present experiment.

It might be meaningless to compare these results directly, because the scrap samples used and the experimental condition are not same. However, comparing the sorting results, it has been found that the calibration method is better than the ANN method.

\section{Conclusion}

The results obtained in this work have demonstrated that the developed LIBS sorting system can classify aluminum alloy scraps into individual alloy groups with a single laser shot and then the classified scrap can be separated into the corresponding container by the sorting signal. It was also found that the sorting algorithm proposed in this work, which utilizes the difference of the concentration of main additional element of aluminum alloy, is reliable and performing.

Finally, practical implementation of the present LIBS sorting system as an on-line tool at the recycling plant requires combining with the feeding system of scrap pieces and the design of automatic feed apparatus which drops the shredded scrap piece into the sequential sample cell is currently in progress.

\section{References}

[1] K. Laqua: "Analytical Laser Spectroscopy", Edited by N. Omenetto, p. 47 (1979), (J. Wiley \& Sons, New York). 
[2] D. A. Cremers, L. J. Radziemski: "Laser-Induced Plasmas and Applications", Edited by L. J. Radziemski, R. W. Solarz and D. A. Cremers, p. 351 (1989), (Marcel Dekker, New York).

[3] J. P. Singh, S. N. Thakur, Laser Induced Breakdown Spectroscopy, (Elsevier Science, Amsterdam, 2007).

[4] P. Werheit, C. Fricke-Begemann, M. Gesing and R. Noll, Journal of Analytical Atomic Spectrometry, 26 (2011) 2166-2174.

[5] J. Gurell, A. Bengtson, M. Falkenström and B. A. M Hansson, Spectrochimica Acta Part B 74-75 (2012) 46-50.

[6] B. Campanella, E. Grifoni, S. Legnaioli, G. Lorenzetti, S. Pagnotta, F. Sorrentino and V. Palleschi, Spectrochimica Acta Part B 134 (2017) 52-57.

[7] M. Kuzuya and O. Mikami, Japanese Journal of Applied Physics, 29 (1990) 1568-1569.

[8] M. Kuzuya, O. Mikami, Journal of Analytical Atomic Spectrometry, 7 (1992) 493-497.

[9] M. Kuzuya, H. Matsumoto, H. Takechi, O. Mikami, Applied Spectroscopy, 47 (1993) 1659-1664.
[10] M. Kuzuya, H. Matsumoto, H. Sakanashi, T. Takemoto, O. Mikami, Journal of Spectroscopical Society of Japan, 43 (1994) 80-84.

[11] M. Kuzuya, J. Tanabe, and K. Suzuki, Journal of Spectroscopical Society of Japan, 46 (1997) 293-296.

[12] M. Kuzuya, H. Aranami, Spectrochimica Acta Part B, 55 (2000) 1423-1430.

[13] M. Kuzuya, M. Murakami, N. Maruyama, Spectrochimica Acta Part B, 58 (2003) 957-965.

[14] T. Yasuda, and M. Kuzuya: Bunseki Kagaku, 54 (2005) 637-641.

[15] B. Chen, M. Kuzuya and T. Yasuda: Journal of Spectroscopical Society of Japan, 55, 191-196 (2006).

[16] B. Chen, H. Kano and M. Kuzuya: Analytical Science, 24 (2008) 289-291.

[17] D. Jiang, H. Kano and M. Kuzuya: Journal of Spectroscopical Society of Japan, 58 (2009) 71-75.

[18] M. Kuzuya, Bunseki Kagaku, 67 (2018) 109-116. 